\title{
Disrupted function and axonal distribution of mutant tyrosyl-tRNA synthetase in dominant intermediate Charcot-Marie-Tooth neuropathy
}

\author{
Albena Jordanova ${ }^{1,2}$, Joy Irobi ${ }^{1}$, Florian P Thomas ${ }^{3,4}$, Patrick Van Dijck ${ }^{5}$, Kris Meerschaert ${ }^{6}$, Maarten Dewil ${ }^{7}$, \\ Ines Dierick ${ }^{1}$, An Jacobs ${ }^{1}$, Els De Vriendt ${ }^{1}$, Velina Guergueltcheva ${ }^{8}$, Chitharanjan V Rao ${ }^{3}$, Ivailo Tournev ${ }^{8}$, \\ Francisco A A Gondim ${ }^{3}$, Marc D’Hooghe ${ }^{9}$, Veerle Van Gerwen ${ }^{1}$, Patrick Callaerts ${ }^{10}$, Ludo Van Den Bosch ${ }^{7}$, \\ Jean-Pièrre Timmermans ${ }^{11}$, Wim Robberecht ${ }^{7}$, Jan Gettemans ${ }^{6}$, Johan M Thevelein ${ }^{5}$, Peter De Jonghe ${ }^{1,12}$, \\ Ivo Kremensky ${ }^{2}$ \& Vincent Timmerman ${ }^{1}$
}

\begin{abstract}
Charcot-Marie-Tooth (CMT) neuropathies are common disorders of the peripheral nervous system caused by demyelination or axonal degeneration, or a combination of both features. We previously assigned the locus for autosomal dominant intermediate CMT neuropathy type C (DI-CMTC) to chromosome 1p34-p35. Here we identify two heterozygous missense mutations (G41R and E196K) and one de novo deletion (153-156delVKQV) in tyrosyl-tRNA synthetase (YARS) in three unrelated families affected with DI-CMTC. Biochemical experiments and genetic complementation in yeast show partial loss of aminoacylation activity of the mutant proteins, and mutations in YARS, or in its yeast ortholog TYS1, reduce yeast growth. YARS localizes to axonal termini in differentiating primary motor neuron and neuroblastoma cultures. This specific distribution is significantly reduced in cells expressing mutant YARS proteins. YARS is the second aminoacyl-tRNA synthetase found to be involved in CMT, thereby linking protein-synthesizing complexes with neurodegeneration.
\end{abstract}

Dominant intermediate CMT neuropathy is a genetic and phenotypic variant of classical CMT characterized by intermediate nerve conduction velocities and histological evidence of both axonal and demyelinating features. Loci have been reported for three DI-CMT types: DI-CMTA has been assigned to chromosome 10q24.1-q25.1 (ref. 1), DI-CMTB to 19p12-p13.2 (ref. 2) and DI-CMTC to 1p34-p35 (ref. 3); in addition, DI-CMTB is known to be caused by mutations in DNM2 (ref. 4). We previously assigned the locus associated with DI-CMTC to a 6.2-cM chromosomal region in two unrelated pedigrees from Bulgaria (CMT-176) and North America (CMT-160). These families do not share a common disease-associated haplotype, indicating that the mutations underlying DI-CMTC arose independently ${ }^{3}$.

In this study, we identified additional recombinants that reduced the genetic interval between SDC3 and D1S2830 to $2.8 \mathrm{Mb}$. In the gene encoding tyrosyl-tRNA synthetase (YARS), we identified a heterozygous transition in exon 2 (G41R) in family CMT-160 and a heterozygous transition in exon 5 (E196K) in family CMT-176. Each missense mutation segregated with DI-CMTC and was absent in 360 European and 262 geographically and ethnically matched Bulgarian control chromosomes. We further screened 255 unrelated individuals with CMT and identified a 12-bp in-frame deletion (153-156delVKQV) in exon 4 in an affected individual from Belgium (PN-765; Fig. 1a). Mutation analysis and genotyping of her asymptomatic parents showed that this mutation occurred de novo.

YARS (also known as TyrRS) catalyzes the aminoacylation of tRNA ${ }^{\text {Tyr }}$ with tyrosine by a two-step mechanism. Tyrosine is first activated by ATP to form tyrosyl-adenylate and is then transferred to tRNA ${ }^{\text {Tyr }}$ (ref. 5). Besides its essential role in protein biosynthesis, YARS has cytokine activities $^{6-8}$. The holoenzyme is a homodimer, in which each monomer comprises two fragments with different biochemical properties (Fig. 1b). The G41R mutation affects an evolutionarily highly conserved glycine residue involved in tyrosyl-adenylate formation

\footnotetext{
${ }^{1}$ Department of Molecular Genetics, Flanders Interuniversity Institute for Biotechnology, University of Antwerp, Universiteitsplein 1, 2610 Antwerpen, Belgium. ${ }^{2}$ Laboratory of Molecular Pathology, Sofia Medical University, 1431 Sofia, Bulgaria. ${ }^{3}$ Department of Neurology, St. Louis University, St. Louis, Missouri 63110, USA. ${ }^{4}$ Department of Molecular Microbiology and Immunology, Institute for Molecular Virology, Spinal Cord Injury Service, St. Louis Veterans Affairs Medical Center, St. Louis University, St. Louis, Missouri 63110, USA. ${ }^{5}$ Department of Molecular Microbiology, Flanders Interuniversity Institute for Biotechnology, Laboratory of Molecular Cell Biology, University of Leuven, 3001 Leuven, Belgium. ${ }^{6}$ Department of Medical Protein Research, Flanders Interuniversity Institute for Biotechnology, Ghent University, 9000 Ghent, Belgium. ${ }^{7}$ Laboratory of Neurobiology, Department of Experimental Neurology, University of Leuven, 3000 Leuven, Belgium.

${ }^{8}$ Department of Neurology, Sofia Medical University, 1431 Sofia, Bulgaria. ${ }^{9}$ Department of Neurology, Sint-Jan Hospital, 8000 Brugge, Belgium. ${ }^{10}$ Laboratory of Developmental Genetics, Flanders Interuniversity Institute for Biotechnology, Departments of Human Genetics and Biology, University of Leuven, 3000 Leuven, Belgium. ${ }^{11}$ Laboratory of Cell Biology and Histology, University of Antwerp, 2020 Antwerpen, Belgium. ${ }^{12}$ Division of Neurology, University Hospital Antwerpen, 2650 Antwerpen, Belgium. Correspondence should be addressed to V.T. (vincent.timmerman@ua.ac.be).
} 

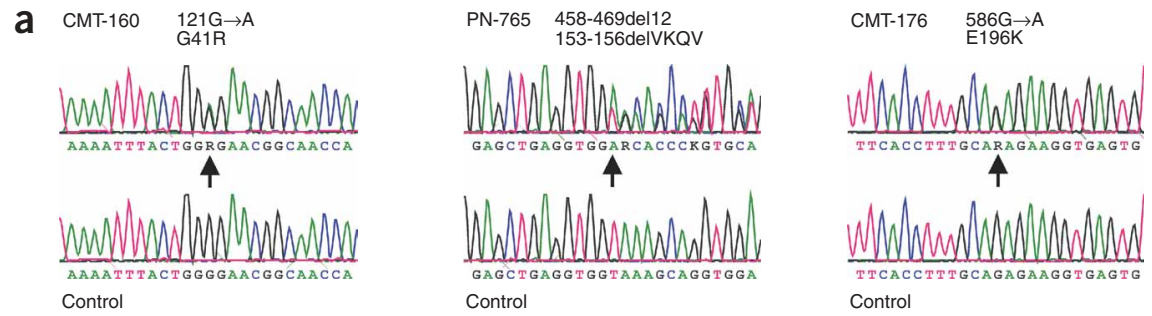

Control

b

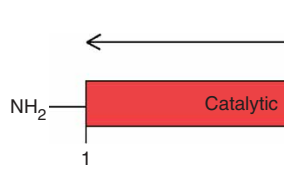

mini TyrRS

EMAPII-like

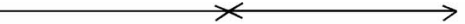

C
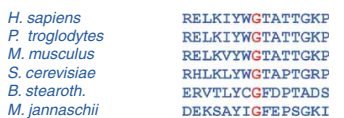

ERVTLYCGED PTAD
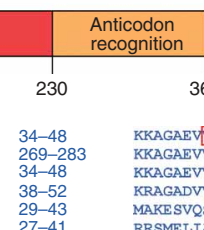

C-terminal

364

KKAGAEY VKQVVEHPI

KKAGAEVVKQVEHP

KKAGAE VVRQVEHP

MAKESVQSRIET--S

$132-146$
$131-145$

Figure 1 Mutations in human YARS associated with DI-CMTC. (a) Electropherograms showing the three sequence variations in YARS. Arrows denote heterozygous mutations. (b) The two principal functional parts of the human TyrRS protein and its corresponding domains. The $\mathrm{N}$ terminus (mini TyrRS) has aminoacylation and interleukin-8-like activity; the $\mathrm{C}$ terminus is homologous to endothelial monocyteactivating polypeptide II (EMAPII). (c) Protein sequence alignment of YARS orthologs. Amino acids involved in CMT-160 and CMT-176 are highlighted in red; amino acids deleted in PN-765 are boxed in red.

(Fig. 1c). Mutations neighboring G41 in bacteria and yeast result in reduced enzymatic activity owing to altered substrate binding ${ }^{9-11}$. The E196 and $\mathrm{V}_{153} \mathrm{KQV}_{156}$ residues are not strictly conserved, but are located in regions that make contact with $\mathrm{tRNA}^{\mathrm{Tyr}}$ (ref. 12 and Fig. 1c).

Because the G41R and E196K mutations are located in the $\mathrm{N}$-terminal part (termed 'mini TyrRS') that contains the aminoacylation domain ${ }^{13}$, we purified recombinant mini TyrRS variants and investigated the effect of both missense mutations on the first step of the aminoacylation reaction. We observed partial loss of activity of both mutant proteins, which was more pronounced for the G41R (34\% residual activity) than for the E196K (53\%) mutant as compared with the wild-type protein (Fig. 2).

To study the functional effects of the mutations in vivo, we carried out genetic complementation experiments in Saccharomyces cerevisiae. We introduced the missense mutations into the yeast ortholog TYS1 (G41R and E196K mutations in YARS correspond to G45R and E200K mutations in TYS1 and are hereafter termed mutG and mutE, respectively). We subsequently transformed a heterozygous deletion strain (TYS1/tys $1:: K A N^{\mathrm{R}}$ ) with wild-type or mutant TYS1 cloned either into a multicopy plasmid (pBEVY) regulated by an $A D H 1$ promoter or into a single-copy plasmid (YCplac111) under its own promoter and terminator. Transformants were sporulated, and the resulting tetrads were dissected to obtain haploid spores with no endogenous expression of TYS1.

We found differences in the number and size of segregant colonies expressing wild-type and mutant TYS1 (Fig. 3a). The mutE protein partially complemented TYS1 deficiency, whereas the mutG protein did not. When TYS1 was expressed under the GAL1 promoter, we observed complementation and small colonies not only for the wild type and mutE proteins, but also for the mutG protein (data not shown), demonstrating that the mutG Tys1 protein has residual activity but is insufficient to support yeast multiplication in the absence of strong overexpression. Because YARS can complement TYS1 deficiency ${ }^{14}$, we carried out similar experiments with the human gene. mutE YARS segregants showed a phenotype similar to that of wild-type YARS segregants, whereas again mutG YARS spores were nonviable, indicating that this mutant protein can not complement TYS1 deficiency (Fig. 3b).

We studied the effect of mutant over wildtype protein by monitoring the growth rate of the TYS1/tys1::KAN ${ }^{\mathrm{R}}$ strain transformed with wild-type TYS1 or YARS and their different mutants under regulation of the conditional GAL1 promoter. We grew cells on selective minimal medium ( $\mathrm{SD}-\mathrm{Ura}$ ), with galactose or glucose as the carbon source to express or suppress the transgene, respectively. All wild-type and mutant TYS1 transformants showed the same growth on glucose-containing medium, whereas on galactose the mutE and mutG TYS1 transformants showed a clear growth delay as compared with transformants expressing wildtype TYS1 (Fig. 3c). The same experiment with YARS showed that expression of mutG YARS significantly reduced cell growth, whereas the mutE YARS yeast culture grew similarly to the wild-type culture (Fig. 3d). This suggested that at least one mutant allele of the human enzyme can be dominant over the wild-type yeast allele. Taken together, these results show that the mutant proteins have a dominant-negative effect on the functionality of the wild-type protein, possibly by lowering its enzymatic activity through dimerization between wild-type and mutant subunits. We verified that heterodimers form between wild-type and mutant YARS isoforms by coimmunoprecipitation (Supplementary Fig. 1 and Supplementary Methods online).

YARS is expressed ubiquitously, including in brain and spinal cord (data not shown). To determine why mutations in YARS result in peripheral neuropathy, we carried out immunofluorescence experiments in fibroblasts (COS1) and neuroblastoma (N2a) cells. We first visualized endogenous expression of YARS in nontransfected cells

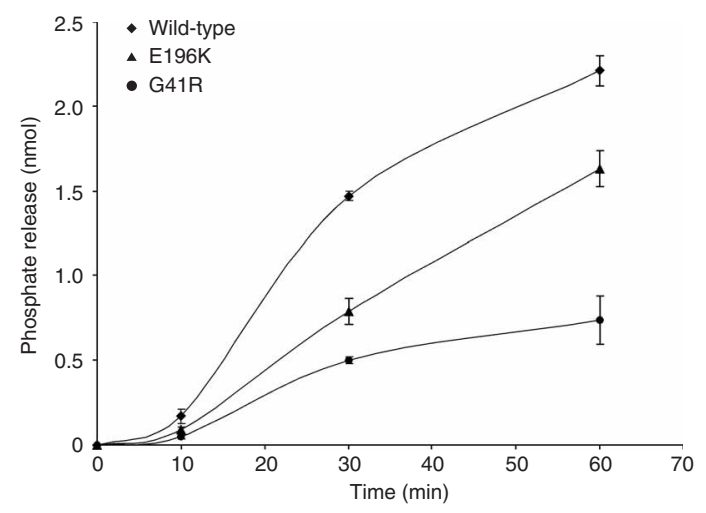

Figure 2 Pyrophosphate release assay. Recombinant mini wild-type, G41R and E196K TyrRS proteins were used to assay the first step of the aminoacylation reaction as described ${ }^{30}$. The pyrophosphate released during amino acid activation is hydrolyzed by pyrophosphatase and the free phosphate is measured in a colorimetric reaction. Data were obtained from three independent experiments. Enzymatic activity was estimated according to the amount of phosphate released after $30 \mathrm{~min}$ of incubation: wild type, $1.47 \pm 0.02 \mathrm{nmol}$; G41R, $0.50 \pm 0.14 \mathrm{nmol}$; E196K, $0.79 \pm 0.10 \mathrm{nmol}$. 
Figure 3 Genetic complementation and growth tests in S. cerevisiae. $(\mathbf{a}, \mathbf{b})$ Tetrad dissection of meiotic segregants of a heterozygous TYS1/ tys $1:: K A N^{R}$ strain transformed with pBEVY_TYS1 or pBEVY_YARS constructs. (a) From each tetrad containing the wild-type TYS1 gene expressed under a constitutive $A D H 1$ promoter (pBEVY_U_TYS1wt), we obtained two colonies with endogenous TYS1 (TYS1 + pBEVY_U_TYS1wt) and two equal-sized colonies with only exogenous TYS1 (tys 1::KANR + pBEVY_U_TYS1wt). The pBEVY_U_TYS1mutE transformants also gave two normal colonies (TYS1 + pBEVY_U_TYS1mutE) and two smaller colonies (tys 1::KANR + pBEVY_U_TYS1mutE). Yeast transformed with the pBEVY_U_TYS1mutG construct yielded only two colonies with endogenous TYS1. Similar results were obtained with a YCplac111 construct (data not shown), showing that the mutE protein partially complements TYS1 deficiency, but the mutG protein does not. (b) From each tetrad containing the wild-type or mutE YARS gene expressed under the conditional GAL1 promoter (pBEVY_GU_YARSwt or pBEVY_GU_YARSmutE), we obtained on SDgal-Ura medium two normalsized colonies with endogenous TYS1 and two small-sized colonies with a null background complemented by exogenous YARS. In the tetrad dissection of tys $1:: K A N^{R}+$ pBEVY_GU_YARSmutG, transformants produced only two normal-sized colonies, and spores containing only the mutG YARS gene were nonviable. Protein blotting showed the presence of mutG protein in cell lysates of heterozygous transformants (TYS1/tys 1::KANR +

pBEVY_GU_YARSmutG), demonstrating that the lack of complementation was not due to a deficiency in protein expression (Supplementary Fig. 3 online and Methods). In all experiments shown in $\mathbf{a}$ and $\mathbf{b}$, complementation of null spores was verified by replica plating on medium selective for the $U R A 3^{+} K A N^{R+}$ phenotype. (c,d) Yeast growth curves of a heterozygous TYS1/ tys 1::KANR strain transformed with pBEVY_GU_TYS1 (c) or pBEVY_GU_YARS (d) constructs containing wild-type and the respective DI-CMTC mutant genes. The strains were grown in SD-Ura media with glucose or galactose as a carbon source.

using an antibody to YARS. Differences in protein distribution were observed between neuronal and non-neuronal cells, and moreover between undifferentiated and differentiating neuronal cells (Fig. 4). Notably, YARS concentrated in granular structures in the growth cone, branch points and the most distal part of projecting neurites in differentiating N2a cells (Fig. 4b-e). A similar distribution along cell projections was found for synaptophysin, a vesicle protein of synaptosomes $^{15}$ (Fig. 4f). This distribution, which we call the 'teardrop effect', was observed in all cells with neurite projections. In cells making extensions, YARS concentrated at the site of outgrowth, behaving like an early polarity marker. YARS localized in a comparable pattern in differentiating human neuronal SH-SY5Y cells (data not shown).

We also examined anti-YARS staining in primary embryonic (E14) motor neurons (Fig. 4i-t). At this relatively early stage of differentiation, YARS was localized throughout the motor neuron and in the small filopodia projecting from the main neurite (Fig. 4, arrows). To test whether this distribution in neuronal cells is characteristic of YARS, we compared it with those of arginyl-tRNA synthetase (ArgRS), an enzyme component of the multi-aminoacyl-tRNA synthetase (AARS) complex ${ }^{16}$, and tryptophanyl-tRNA synthetase (TrpRS), a structural homolog of YARS and a molecular antagonist of its cell signaling function ${ }^{17}$. None of these AARSs showed the same teardrop effect (Fig. 4g,h), suggesting that YARS has a specific role in neuronal endings where it is predominantly expressed.
To search for neuron-specific defects, we transiently transfected N2a cells with pEGFP constructs expressing wild-type and mutant YARS proteins (Fig. 5). At 7-72 h after transfection, the distribution of YARS in cells transfected with a wild-type (Fig. 5a-c) or control (data not shown) vector was comparable to that observed in nontransfected cells (Fig. 5b,c). In cells expressing mutE and mutG proteins, however, the teardrop effect was reduced and YARS was homogeneously distributed in most cells without preferential sorting to neuronal tips (Fig. $\mathbf{5 d - i}$ ). In addition, there was no significant difference in the localization of synaptophysin in mutant cells, and the distribution of mitochondria along neuronal outgrowths was preserved (Supplementary Fig. 2 online). Because many mRNAs targeted to the nerve terminals are transported along microtubules ${ }^{18}$, we also stained for $\alpha$ - and $\beta$-tubulin. Although the microtubular network was intact, suggesting that mutant YARS does not interfere with network formation, disrupted interactions with other proteins remain plausible (Supplementary Fig. 2).

We have identified mutations in YARS that cause DI-CMT in three unrelated families. YARS is the second AARS known to be associated with inherited peripheral neuropathies. Dominant mutations in GARS, which encodes glycyl-tRNA synthetase, have been found in CMT2D and in distal spinal muscular atrophy type V, two allelic neuropathies assigned to chromosome 7p14 (ref. 19). In addition, p38, a subunit of the AARS complex, is a parkin substrate in a model of 

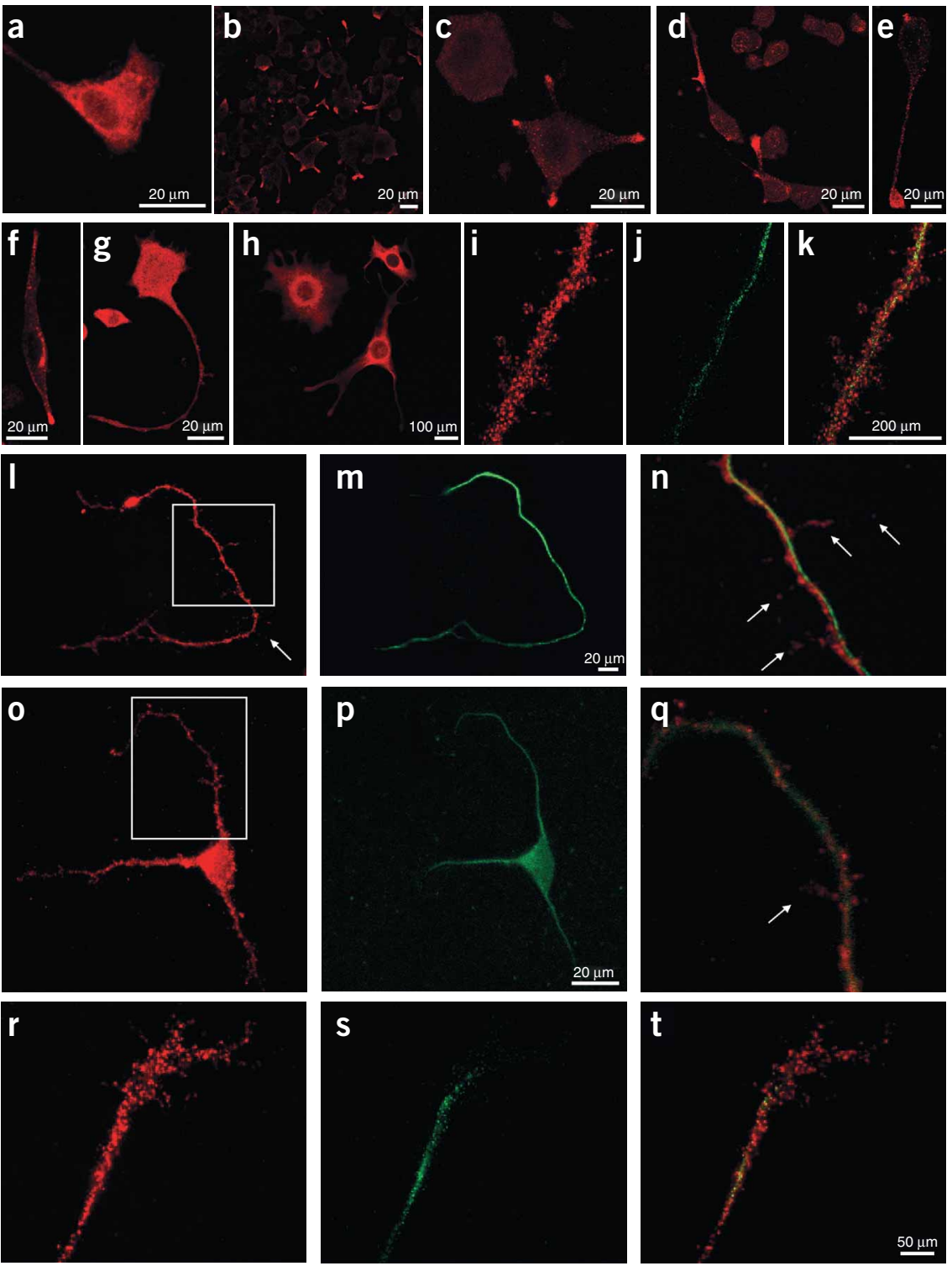

Figure 4 Cellular distribution of aminoacyl-tRNA synthetases, synaptophysin and neurofilaments. (a) Homogeneous localization of YARS throughout the cytoplasm in COS1 cells. (b) Representative image of differentiating N2a cells expressing YARS. (c) Nondifferentiated N2a cell (left) with homogenous expression pattern and differentiating N2a cell (right) with polarized distribution of YARS along the neurite-forming part of the cell. (d) Expression of YARS in the branch point and growing processes.

(e-h) Localization of YARS in the growth cone (e), compared with the distribution of synaptophysin (f), ArgRS (g) and TrpRS (h) in differentiating N2a cells. (i-s) Immunostaining of both YARS $(\mathbf{i}, \mathbf{l}, \mathbf{0}, \mathbf{r})$ and nonphosphorylated neurofilaments $(\mathbf{j}, \mathbf{m}, \mathbf{p}, \mathbf{s})$ in primary motor neurons of E14 mice. $\mathbf{k}, \mathbf{n}, \mathbf{q}$ and $\mathbf{t}$ show colocalization. $\mathbf{n}$ and $\mathbf{q}$ represent the boxed areas in I and $\mathbf{o}$, respectively. Note the granular staining of YARS along the neurite projections, filopodia (arrow) and growth cone (r). SMI32 staining was used as a marker to identify motor neurons and their neurites.

Because embryonic cultures were used, the signal is weak $(\mathbf{j}, \mathrm{m}, \mathrm{p}, \mathbf{s})$.

necessary proteins. Nerve endings partially function independently from the cell body and contain mRNAs, ribosomes, AARSs and other co-translational machinery (see references in ref. 21). Protein synthesis requires the presence of all 20 AARSs; however, the specific subcellular distribution of YARS was not observed for two other AARSs (ArgRS and TrpRS), at least in our experiments. ArgRS has been shown to colocalize with the extrasomatic translational complexes ${ }^{22}$, and we have shown that ArgRS is not enriched in the neuronal terminals. This suggests that YARS might have an additional axon-terminal-specific function.

Inherited peripheral neuropathies are caused by length-dependent axonal degeneration, which starts in the most distal parts of

Parkinson's disease and shows particularly intense immunoreactivity in dopaminergic neurons ${ }^{20}$. These findings support the notion that AARSs and other protein-synthesizing complexes may be involved in neurodegeneration.

Our genetic complementation and growth assays in yeast and biochemical data provide evidence that mutations associated with DI-CMTC alter the normal properties of YARS. These mutations reduce the first step of the aminoacylation reaction and have a dominant-negative effect on wild-type function. Notably, the G41R mutation has a more severe effect, corresponding to $66 \%$ loss of enzymatic activity and lack of functional complementation in TYS1deficient yeast spores. This underscores the importance of this conserved glutamate residue and correlates with the more severe phenotype of the individuals in the North American family affected with DI-CMTC. The connection between the DI-CMTC phenotype and the canonical function of YARS remains, however, unknown. Our immunocytochemical experiments showed that YARS localizes to axonal termini. YARS could be a component of the protein-synthesizing complexes in projecting neurons, providing the leading edge with the nerves and spreads towards the cell body. The neuron is the longest and most polarized type of cell, requiring an efficient axonal transport of cytoskeletal and synaptic vesicle components, mitochondria and other cellular organelles. An increasing number of disease-associated genes in CMT and related neuropathies are being found to have, despite their pleiotropic functions, a major effect on axonal transport in the peripheral nervous system ${ }^{23}$. Our results are in accordance with these findings and stress the importance of local metabolism in synaptosomes and neuronal endings as the possible site where the disease pathology starts. Studies on rat dorsal root ganglia and motor neurons have shown that intra-axonal translation facilitates their regeneration processes ${ }^{24}$, and that inhibition of local protein synthesis reduces the regeneration rate of adult rat peroneal nerve ${ }^{25}$. Our combined genetic and functional experiments show that YARS mutations causing DI-CMTC have a dominant-negative effect on the normal function and distribution of YARS in neuronal endings, which in turn could affect synaptic plasticity and result in axonal degeneration, leading to axonal loss and ultimately to a motor and sensory peripheral neuropathy. 
Figure 5 Confocal images of N2a cells transiently transfected with wild-type and mutant YARS constructs. N2a cells were transfected with the constructs pEGFP-C1_YARSwt (a-c), pEGFPC1_YARSmutE (d-f) and pEGFP-C1_YARSmutG $(\mathrm{g}-\mathrm{i})$ and examined for green fluorescence $7 \mathrm{~h}$ after transfection $(\mathbf{a}, \mathbf{d}, \mathbf{g})$. The same cells were processed for red fluorescent immunostaining with antibody to YARS (b,e,h). Merged images are shown in $\mathbf{c}, \mathbf{f}$ and $\mathbf{i}$, respectively. Scale bars, $20 \mu \mathrm{m}$. The percentage of cells showing the 'teardrop effect' among nontransfected cells was $73 \pm 8 \%$, which is similar to that of pEGFPtransfected cells $(67 \pm 5 \%)$. This teardrop distribution was significantly reduced in cells expressing mutE $(29 \pm 6 \%)$ and mutG $(27 \pm 7 \%)$, as compared with cells expressing wild-type YARS $\left(61 \pm 9 \% ; P=1.3 \times 10^{-3}\right.$ and $P=1.2$ $\times 10^{-3}$ respectively; data from four independent experiments). Protein blot analysis of protein extracts obtained from $\mathrm{N} 2 \mathrm{a}$ and $\mathrm{COS} 1$ cells transfected with the wild-type and mutant YARS constructs showed comparable expression of the recombinant proteins (Supplementary Fig. 3).
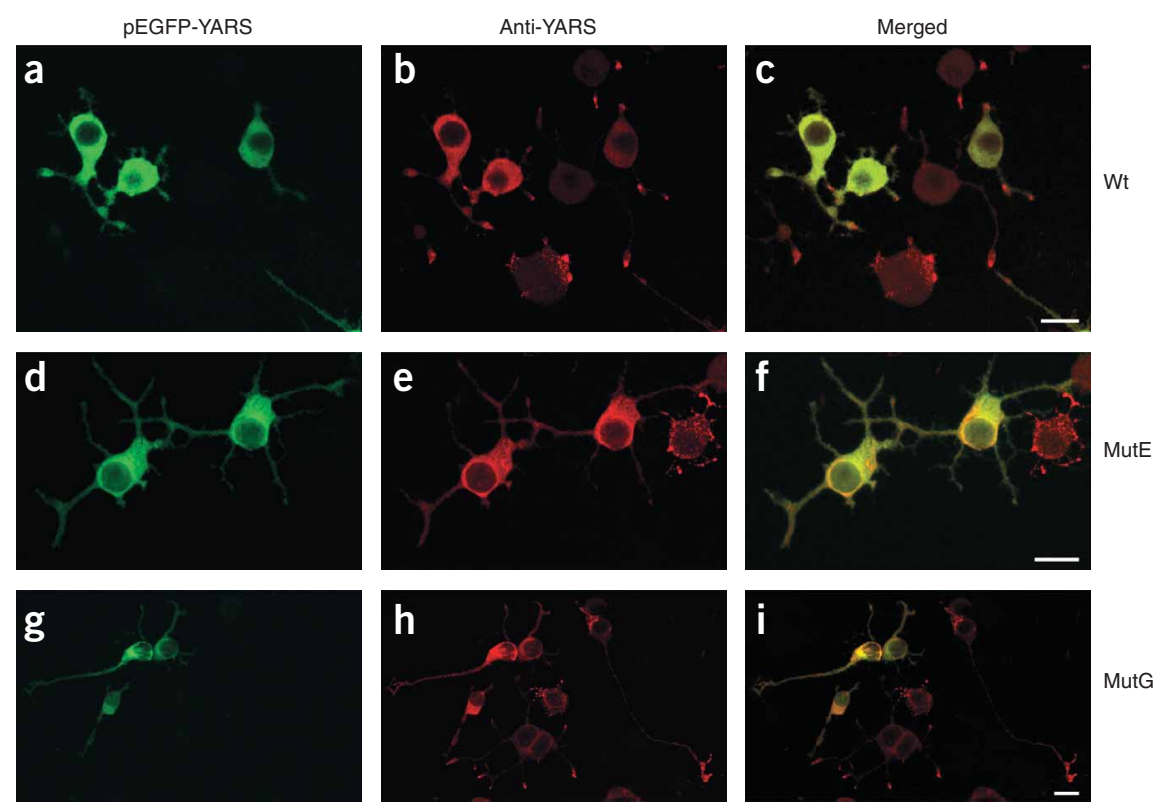

\section{METHODS}

DI-CMTC families. The study included two families with DI-CMTC previously linked to 1p34-p35: a North American (CMT-160) and a Bulgarian (CMT-176) family ${ }^{3}$. Individuals affected in both families show a motor and sensory neuropathy with onset between age 10 and 60 years, distal weakness and leg atrophy, motor median nerve conduction velocities between 25 and $58 \mathrm{~m} / \mathrm{s}$, slow progression and moderate severity. Interfamilial comparisons showed that individuals affected in the North American family have an earlier onset, more sensory abnormalities and lower nerve conduction velocities. We also screened the YARS gene for mutations in 127 individuals with demyelinating, 16 with intermediate and 112 with axonal CMT neuropathies. From all family members and control individuals, genomic DNA was isolated from peripheral blood by a standard procedure. Paternity was confirmed in family PN-765. Informed consent was obtained from all family members and the study was approved by the Institutional Review Boards of the Universities of Antwerp, Sofia and St. Louis.

Mutation analysis. We used the National Center for Biotechnology Information (NCBI) Entrez Genome Map Viewer, the Ensembl Human Genome Server and GenBank to search for known genes, expressed sequence tags and putative genes in the DI-CMTC region. We determined exon-intron boundaries by BLAST searches against the high-throughput genome sequences (NT_004511). From the refined region, containing 37 known and 19 predicted genes, we excluded by DNA sequencing ten selected genes (CSMD2, FABP3, FNDC5, WDR57, MARCKSL1, PEF1, PUM1, RBBP4, SYNC1 and ZNF31), and subsequently found three mutations in the gene YARS. All coding exons and exon-intron boundaries of candidate genes were sequenced as described ${ }^{3}$. We based codon numbering of YARS on the reported amino acid sequence, using the first methionine as an initiation codon (NCBI accession numbers NP_003671 and NM_003680).

cDNA cloning and mutagenesis. For yeast experiments, we used the multicopy pBEVY plasmid and the single-copy YCplac111 plasmid. Full-length YARS cDNA was derived by PCR using the RZPD clone IMAGp998G1110070Q as a template. The TYS1 sequence was amplified from yeast genomic DNA. For expression in mammalian cells, full-length wild-type or mutant YARS cDNAs were cloned into pEGFP-C1 (Clontech) or pcDNA3.1/V5His-TOPO (Invitrogen) vectors. The mini TyrRS cDNA sequence, encompassing amino acids 1-364, was cloned into the pCR-T7/NT-TOPO-TA vector (Invitrogen). For site-directed mutagenesis of YARS and TYS1, we used the QuickChange kit (Stratagene). All constructs were verified by sequencing.
RNA blot analysis. RNA blotting was done by using a custom-made human brain blot and the FirstChoice Human Blot 1 (Ambion) according to the manufacturer's protocol. As a probe, we used a gel-purified 2.5-kb YARS cDNA fragment labeled with $\left[\alpha^{32} \mathrm{P}\right]$-dCTP by the Random Primers DNA labeling system (Invitrogen).

Cell cultures, growth and transfection conditions. Mouse (N2a) and human (SH-SY5Y) neuroblastoma cell lines were maintained in minimal essential medium; HEK293T cells and monkey kidney fibroblast (COS1) cells were maintained in Dulbecco's modified Eagle's medium supplemented with $10 \%$ fetal bovine serum at $37{ }^{\circ} \mathrm{C}$ and $5 \% \mathrm{CO}_{2}$. Cells were grown on $4-\mathrm{cm}^{2}$ surface plates and transfected with $0.5 \mu \mathrm{g}$ of plasmid DNA using Lipofectamine 2000 (Invitrogen). N2a cells were differentiated by incubation in media without serum for $72 \mathrm{~h}$. We differentiated SHY-5Y cells as described ${ }^{26}$. Motor neuron cultures were prepared as described ${ }^{27}$.

Yeast strain, growth and tetrad analysis. We used S. cerevisiae strain Y24815 (MATa/alpha his3/his3 leu2/leu2 ura3/ura3 met15/MET15 LYS2/lys2 TYS1/tys1:: KAN ${ }^{\mathrm{R}}$ ) derived from the Euroscarf collection (Invitrogen). Yeasts were grown in rich (YP) or minimal (SD) medium supplemented with glucose (SDglu) or galactose (SDgal). For selection of kanamycinresistant spores, yeasts were grown on YP plates containing G-418 (Duchefa). The yeast strain was transformed with plasmid DNA by using lithium acetate, and transformants were cultured on SDglu or SDgal media. Tetrad analysis was done by standard procedures. At least 18 tetrads per transformant were dissected with a micromanipulator (Singer Instruments). Spores were germinated on rich or minimal medium at $30{ }^{\circ} \mathrm{C}$, and their genotype was determined by replica plating on SDglu or SDgal medium lacking leucine or uracil and on YPD or YPgal medium plus Geneticin. We measured growth measurements as described ${ }^{28}$. All yeast cultures were monitored in triplicate.

Production and purification of recombinant mini TyrRS. Expression of wild-type and mutant mini TyrRS was induced with $1 \mathrm{mM}$ isopropyl- $\beta$-Dthiogalactopyranoside for $3 \mathrm{~h}$ at $37^{\circ} \mathrm{C}$. Cultures were lysed in $50 \mathrm{mM}$ Tris$\mathrm{HCl}(\mathrm{pH} 7.5), 100 \mathrm{mM} \mathrm{KCl}, 5 \mathrm{mM} \beta$-mercaptoethanol, protease inhibitor cocktail, $1 \mathrm{mM}$ phenyl methylsulfonyl fluoride and $1 \%$ Nonidet P-40. After centrifugation for $30 \mathrm{~min}$ at $100,000 \mathrm{~g}$, the supernatant was incubated with Probound resin (Invitrogen), and bound proteins were eluted with imidazole and analyzed by SDS-PAGE. Protein concentration was measured by using bovine serum albumin as a standard. 
Pyrophosphate release assay. Pyrophosphate release was assayed at $37{ }^{\circ} \mathrm{C}$ in $50 \mu \mathrm{l}$ of reaction buffer containing $100 \mathrm{mM}$ Tris- $\mathrm{HCl}(\mathrm{pH} 7.6), 10 \mathrm{mM} \mathrm{MgCl}_{2}$, $40 \mathrm{mM} \mathrm{KCl}, 1 \mathrm{mM}$ dithiothreitol, $40 \mathrm{U} / \mathrm{ml}$ of bovine liver tRNA (Sigma), $5 \mathrm{U} / \mathrm{ml}$ of yeast inorganic pyrophosphatase (Sigma), $1 \mathrm{mM}$ ATP and $0.2 \mathrm{mM}$ L-tyrosine. Purified recombinant mini wild-type and mutant TyrRS proteins $(1 \mu \mathrm{M})$ were assayed for 0-60 min, Biomol Green reagent (Biomol) was added to the mixture and absorbency at $620 \mathrm{~nm}$ was measured using a Multiscan EX microplate reader (Thermo Labsystems). Spontaneous hydrolysis of ATP was negligible in the assay conditions used.

Immunocytochemistry and microscopy. Cellular EGFP fluorescence was analyzed after fixation with $3 \%$ paraformaldehyde in phosphate-buffered saline for $25 \mathrm{~min}$ at $22{ }^{\circ} \mathrm{C}$. Alternatively, cells were processed for single and double immunolabeling ${ }^{29}$ at $7,24,48$ and $72 \mathrm{~h}$ after transfection. We used primary antibodies against TyrRS (1:25 dilution; C-18 from Santa Cruz), ArgRS (1:50) and TrpRS (1:50; both from L. Kleiman, Institute for Medical Research, Montreal, Canada), $\alpha$-tubulin (1:100; Sigma), $\beta$-tubulin (1:50; E7), synaptophysin (1:50; Transduction Laboratories), nonphosphorylated neurofilaments (1:100; SMI32 from Sternberger Monoclonal) and $\beta$-actin (1:5,000; Sigma). For secondary antibodies, we used donkey anti-goat, antimouse and anti-rabbit IgGs conjugated to Alexa Fluor 594, 555 or 488 (Molecular Probes). MitoTracker Red $\mathrm{CM}-\mathrm{H}_{2} \mathrm{Xros}$ (Molecular Probes) was used as a mitochondrion-selective dye. Cells were examined by a Zeiss LSM510 confocal microscope equipped with Meta-Analysis tool and SP2v.3.2 software (Zeiss).

Accession numbers. GenBank protein sequences for YARS/TyrRS: Homo sapiens, NP_003680; Pan troglodytes, XP_5246511; Mus musculus, NP_598912; S. cerevisiae, NP_011701; Bacillus stearothermophilus, P00952; Methanococcus jannaschii, NP_247363. The genomic sequence NT_004511 includes the genes FABP3 (NM_004102), FNDC5 (NM_004814), WDR57 (NM_004814), CSMD2 (NM_052896), MARCKSL1 (NM_023009), PEF1 (NM_012392), PUM1 (NM_014676), RBBP4 (NM_005610), SYNC1 (NM_030786) and ZNF31 (NM_145238).

URLs. Genome Database, http://www.gdb.org/; National Center for Biotechnology Information, http://www.ncbi.nlm.nih.gov/; Entrez Genome Map Viewer, http://www.ncbi.nlm.nih.gov/mapview/; Ensembl Human Genome Server, http://www.ensembl.org/; GenBank, http://www.ncbi.nlm.nih.gov/ entrez/query.fcgi?db=Nucleotide; RZPD, http://www.rzpd.de/; ClustalW multiple protein alignment software, http://npsa-pbil.ibcp.fr/cgi-bin/npsa_automat. pl?page=/NPSA/npsa_clustalw.html.

\section{Note: Supplementary information is available on the Nature Genetics website.}

\section{ACKNOWLEDGMENTS}

We acknowledge the cooperation and participation of all patients and their relatives in this study. We thank the VIB Genetic Service Facility for genotyping and sequencing; C. Van Broeckhoven for support; V. De Corte and D. Adriaensen for discussions; J. Van Daele and D. De Rijck for assistance with confocal microscopy; C. Coun, C. Colombo and E. Vanderheyden for help with the yeast experiments; and B. Ishpekova and I. Litvinenko for clinical evaluation of the patients. This study was supported by the National Science Fund of the Bulgarian Ministry of Education and Science; the Fund for Scientific Research - Flanders (FWO); the Medical Foundation Queen Elisabeth; the Universities of Antwerp, Leuven and Ghent; and the Interuniversity Attraction Poles program of the Belgian Federal Science Office (POD). Additional support was provided by the Muscular Dystrophy Association (to A.J. and V.T.), the Neuropathy Association (to F.P.T.), the Association Belge contre les Maladies Neuromusculaires (to V.T.) and the Specific Support Action program of the European Union (to A.J. and I.K.). A.J. received visiting research fellowships from the POD and the North Atlantic Treaty Organisation/FWO. J.I. and K.M. are postdoctoral fellows of the FWO, and I.D. is a PhD fellow of the Institute for Science and Technology, Belgium.

\section{COMPETING INTERESTS STATEMENT}

The authors declare that they have no competing financial interests.
Published online at http://www.nature.com/naturegenetics

Reprints and permissions information is available online at http://npg.nature.com/ reprintsandpermissions/

1. Verhoeven, K. et al. Localization of the gene for the intermediate form of CharcotMarie-Tooth to chromosome 10q24.1-q25.1. Am. J. Hum. Genet. 69, 889-894 (2001).

2. Kennerson, M.L. et al. Dominant intermediate Charcot-Marie-Tooth neuropathy maps to chromosome 19p12-p13.2. Am. J. Hum. Genet. 69, 883-888 (2001).

3. Jordanova, A. et al. Dominant intermediate Charcot-Marie-Tooth type C maps to chromosome 1p34-p35. Am. J. Hum. Genet. 73, 1423-1430 (2003).

4. Zuchner, S. et al. Mutations in the pleckstrin homology domain of dynamin 2 cause dominant intermediate Charcot-Marie-Tooth disease. Nat. Genet. 37, 289-294 (2005).

5. Fersht, A.R. et al. Active site titration and aminoacyl adenylate binding stoichiometry of aminoacyl-tRNA synthetases. Biochemistry 14, 1-4 (1975).

6. Wakasugi, K. \& Schimmel, P. Highly differentiated motifs responsible for two cytokine activities of a split human tRNA synthetase. J. Biol. Chem. 274, 23155-23159 (1999).

7. Kleeman, T.A., Wei, D., Simpson, K.L. \& First, E.A. Human tyrosyl-tRNA synthetase shares amino acid sequence homology with a putative cytokine. J. Biol. Chem. 272 14420-14425 (1997).

8. Wakasugi, K. \& Schimmel, P. Two distinct cytokines released from a human aminoacyltRNA synthetase. Science 284, 147-151 (1999).

9. Winter, G., Fersht, A.R., Wilkinson, A.J., Zoller, M. \& Smith, M. Redesigning enzyme structure by site-directed mutagenesis: tyrosyl tRNA synthetase and ATP binding. Nature 299, 756-758 (1982).

10. Ohno, S., Yokogawa, T. \& Nishikawa, K. Changing the amino acid specificity of yeast tyrosyl-tRNA synthetase by genetic engineering. J. Biochem. 130, 417-423 (2001).

11. Wilkinson, A.J., Fersht, A.R., Blow, D.M. \& Winter, G. Site-directed mutagenesis as a probe of enzyme structure and catalysis: tyrosyl-tRNA synthetase cysteine-35 to glycine-35 mutation. Biochemistry 22, 3581-3586 (1983).

12. Nair, S. et al. Species-specific tRNA recognition in relation to tRNA synthetase contact residues. J. Mol. Biol. 269, 1-9 (1997).

13. Yang, X.L., Skene, R.J., McRee, D.E. \& Schimmel, P. Crystal structure of a human aminoacyl-tRNA synthetase cytokine. Proc. Natl. Acad. Sci. USA 99, 15369-15374 (2002).

14. Wakasugi, K., Quinn, C.L., Tao, N. \& Schimmel, P. Genetic code in evolution: switching species-specific aminoacylation with a peptide transplant. EMBO J. 17, 297-305 (1998).

15. Sudhof, T.C. The synaptic vesicle cycle: a cascade of protein-protein interactions. Nature 375, 645-653 (1995).

16. Quevillon, S., Robinson, J.C., Berthonneau, E., Siatecka, M. \& Mirande, M. Macromolecular assemblage of aminoacyl-tRNA synthetases: identification of protein-protein interactions and characterization of a core protein. J. Mol. Biol. 285, 183-195 (1999).

17. Yang, X.L., Schimmel, P. \& Ewalt, K.L. Relationship of two human tRNA synthetases used in cell signaling. Trends Biochem. Sci. 29, 250-256 (2004).

18. Olink-Coux, M. \& Hollenbeck, P.J. Localization and active transport of mRNA in axons of sympathetic neurons in culture. J. Neurosci. 16, 1346-1358 (1996).

19. Antonellis, A. et al. Glycyl tRNA synthetase mutations in Charcot-Marie-Tooth disease type 2D and distal spinal muscular atrophy type V. Am. J. Hum. Genet. 72, 12931299 (2003).

20. Corti, O. et al. The p38 subunit of the aminoacyl-tRNA synthetase complex is a Parkin substrate: linking protein biosynthesis and neurodegeneration. Hum. Mol. Genet. 12, 1427-1437 (2003).

21. Giuditta, A., Kaplan, B.B., van Minnen, J., Alvarez, J. \& Koenig, E. Axonal and presynaptic protein synthesis: new insights into the biology of the neuron. Trends Neurosci. 25, 400-404 (2002).

22. Barbarese, E. et al. Protein translation components are colocalized in granules in oligodendrocytes. J. Cell Sci. 108, 2781-2790 (1995).

23. Suter, U. \& Scherer, S.S. Disease mechanisms in inherited neuropathies. Nat. Rev. Neurosci. 4, 714-726 (2003).

24. Zheng, J.Q. et al. A functional role for intra-axonal protein synthesis during axonal regeneration from adult sensory neurons. J. Neurosci. 21, 9291-9303 (2001).

25. Gaete, J., Kameid, G. \& Alvarez, J. Regenerating axons of the rat require a local source of proteins. Neurosci. Lett. 251, 197-200 (1998).

26. Encinas, M. et al. Sequential treatment of SH-SY5Y cells with retinoic acid and brain-derived neurotrophic factor gives rise to fully differentiated, neurotrophic factordependent, human neuron-like cells. J. Neurochem. 75, 991-1003 (2000).

27. Van Damme, P., Callewaert, G., Eggermont, J., Robberecht, W. \& Van den Bosch, L. Chloride influx aggravates $\mathrm{Ca}^{2+}$-dependent AMPA receptor-mediated motoneuron death. J. Neurosci. 23, 4942-4950 (2003).

28. Tanghe, A. et al. Aquaporin expression correlates with freeze tolerance in baker's yeast, and overexpression improves freeze tolerance in industrial strains. Appl. Environ. Microbiol. 68, 5981-5989 (2002).

29. De Corte, V. et al. Increased importin- $\beta$-dependent nuclear import of the actin modulating protein CapG promotes cell invasion. J. Cell Sci. 117, 5283-5292 (2004).

30. Kiga, D. et al. An engineered Escherichia coli tyrosyl-tRNA synthetase for site-specific incorporation of an unnatural amino acid into proteins in eukaryotic translation and its application in a wheat germ cell-free system. Proc. Natl. Acad. Sci. USA 99, 9715-9720 (2002). 